\title{
Design of a new steering system to solve the problem of steering operation during low speed driving
}

\author{
Kei KITAHARA*, Keisuke KAZAMA* and Hiroshi MOURI* \\ * Dept. of Mechanical System Engineering, Tokyo Univ. of Agriculture and Technology \\ 2-24-16 Naka-cho, Koganei-shi, Tokyo 184-8588, Japan \\ E-mail: s180228r@st.go.tuat.ac.jp
}

Received: 30 August 2018; Revised: 12 February 2019; Accepted: 7 May 2019

\begin{abstract}
This study aims to develop a new steering system that can help increase the ease of driving and reduce the driver's mental workload during low-speed driving. First, the problems associated with low-speed driving were investigated. It was found that the act of changing the steering grip is one of the major factors that increase the workload. Therefore, it a new steering system, which eliminates the need of changing the steering grip, should be developed. A preliminary lab experiment was conducted to determine the specifications for the new steering system. Resultantly, it was found that hassle-free low-speed driving can be performed by changing the directions of the driver's arm motions and form of the steering grip, which are the characteristics of a conventional steering system. Therefore, we decided the bar type steering as a proposal system which changed them. A driving simulator experiment was conducted to compare the differences between conventional steering system and the proposed steering system. It was also found that the proposed steering system has a lower driver gain and higher time constant in comparison with a conventional steering system. The proposed steering system enables a hassle-free driving experience, even with a low steering ratio. Finally, an experiment with test subject drivers in a real car was conducted to evaluate the compatibility between the proposed steering system and real car. Furthermore, it was found that the proposed steering system was easy to drive with, even for drivers with no previous driving experience.
\end{abstract}

Keywords : Steering system, Steer-by-wire, Human-machine-interface, Upper limb motion, Driver characteristics

\section{Introduction}

While driving a car, three tasks, which are cognition, decision, and maneuver, are performed in parallel. According to the attentional resources allocation model, a person possesses a fixed and limited amount of attentional resources and performs multiple tasks in parallel using these attentional resources by processing the information regarding these tasks (Kahneman et al., 1973). Additionally, the information processing accuracy of each task is determined by the amount of attentional resources allocated. When the workload for one of the tasks, i.e., cognition, decision, and maneuver, is increased, there is an increase in the amount of information that needs to be processed. Furthermore, human errors and accidents become more likely when the total amount of information that needs to be processed exceeds the driver's attentional resource capacity. Therefore, with an aim of reducing the work required for processing information, a significant amount of research has been conducted on driver warning systems and driving systems. Souma et al. proposed a cognition assistance system using a lane-departure warning that takes the driver's reaction time into account (Souma et al., 2001). Some studies (Sadano et al., 2001; Ishida, 2006) have also proposed methods that can reduce maneuvering workload. However, most of these studies are based on correcting human errors and not based on preventing human error. Furthermore, all these studies are based on high-speed driving. Most of the driving required for daily tasks, such as shopping and commuting, is done at low-speeds, e.g., navigating through intersections, narrow roads, and parking. Low-speed driving typically takes place in a complex driving environment, such as passing other cars, merging, and paying attention to pedestrians, among others, and thus the amount of information processing 
resource allocation required for cognition and decision is high. The maneuvering workload is also increased for low-speed driving due to the high number and complexity of maneuvers. Meanwhile, $\sim 54 \%$ of accidents take place at intersections. If accident locations are broadly categorized as urban and suburban, urban accidents account for $\sim 75 \%$ of all accidents. These facts demonstrate the high risks associated with low-speed driving (National Police Agency Department of Transportation, 2014). However, an efficient method for hassle-free low-speed driving has not been proposed yet.

First, the difficulties faced during low-speed driving are investigated through driver steering action analysis. Then, as a scheme to mitigate the problems identified in the analysis, a new steering system, which reexamines the direction of the driver's arm motions and steering wheel grip form, is proposed. In the proposed study, multiple steering system specifications were prepared, and then a preliminary lab experiment and driving simulator experiment were conducted. An experiment to verify the differences in driver characteristics between the conventional and proposed steering systems was also conducted. Finally, the proposed system was installed on a real car, and then its advantages over conventional steering systems were verified through a subject experiment.

\section{Steering action characterization}

The mechanics of the steering action were recorded and analyzed to identify the problems associated with driving at low-speeds. Steering wheel grip position, steering wheel angle, yaw rate, and yaw angle were used for the steering action performance metrics.

\subsection{Design of the grip position measurement device}

A grip position measurement device was proposed to record the steering wheel grip position, which sometimes changes when steering. Fig. 1 shows the device construction and a partial section view. Pressure sensors with pressure sensitivity ranging between 0.2 and $20 \mathrm{~N}$ were placed (Interlink Electronics) at intervals of 7.5[deg] along the steering wheel circumference. The grip position was determined by the change in surface pressure on the steering wheel. Hall ICs with a sensitivity of $5 \mathrm{mV} / \mathrm{G}$ (MicroSystems) were placed at intervals of $7.5[\mathrm{deg}]$ along the steering wheel circumference and were used to distinguish between the left and right hands. A Hall IC is a non-contacting magnetic sensor, which uses the Hall element to detect magnetic field strength and variation. The driver was asked to wear rings with opposite polarities attached on their left and right hands, as shown in Fig. 2. By reversing the direction of the magnetic flux directed to the steering wheel surface, the direction of the magnetic flux entering the Hall IC is changed. As a result, we can judge right and left of hand.

The hand grip data were collected using a microcomputer that was installed to rotate with the steering wheel and transmit wirelessly to the recording device. A $2.4 \mathrm{GHz}$ band wireless communication module (Digi International) was used for communication to achieve a wireless communication between the steering wheel and recording device. The steering wheel angle was measured using a rotary encoder (Omron) with a resolution of 0.05[deg], and the yaw rate was measured using a gyroscopic sensor with a detection range of \pm 200 [deg/s] (TAMAGAWA Seiki Co. Ltd.). This setup allowed the left and right hand grip positions on the steering wheel to be recorded during maneuvers without having the wheel feel unnatural to the driver.

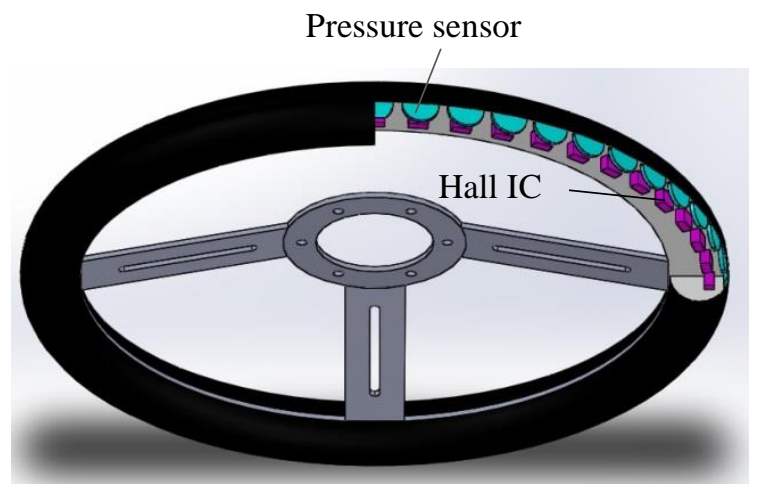

Fig. 1 Partial view of the steering wheel grip position measurement device. The position of the steering wheel grip was determined using pressure sensors and Hall ICs. 


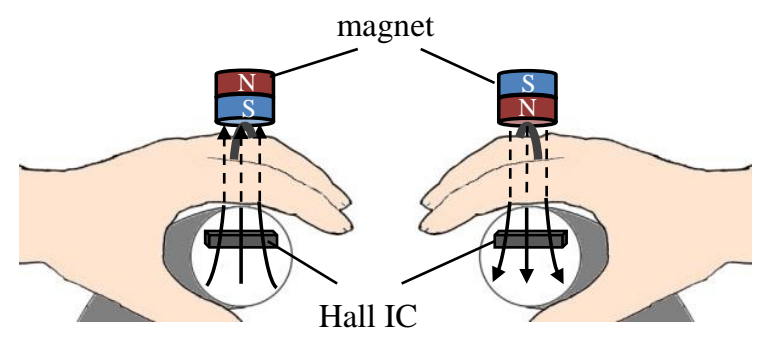

Fig. 2 The test subject wore rings fitted with magnets. The $\mathrm{N}$ pole is facing down for the ring worn on the right hand, while the ring worn on the left hand has the S-pole facing down. This reverses the magnetic flux that crosses the Hall IC between the left and right rings and shows the difference in measured values for the right and left hands.

\subsection{Experimental method}

The test subjects were asked to wear magnetic rings and drive through a test course that emulated a left turn at an intersection, as shown in Fig. 3. The car speed was maintained at $10 \mathrm{~km} / \mathrm{h}$ by driver pedal control. The test subjects were instructed to drive with firm grips on the steering wheel without sliding their hands on the steering wheel and to not temporarily stop and signal their turn at the intersection. The test subjects included three experienced and three inexperienced drivers. After a few practice runs, each driver drove on the course nine times. An experienced driver was defined as someone who drives a car by commuting or going to school in daily life and over 5 years have passed since got driver's license for experienced drivers. An inexperienced driver was defined as someone who has had almost not driving experience since acquiring their driving license (referred to in Japan as "Paper Drivers").

Prior to the experiment, the test subjects were made sufficiently aware of what their participation in the study entailed. More specifically, the research purpose, content, foreseeable risks, possible discomfort, management of personal information, and their right to withdraw from the experiment at any time were explained in detail. Written consent was also obtained. All other experiments detailed in this report have also been subject to similar informed consent.

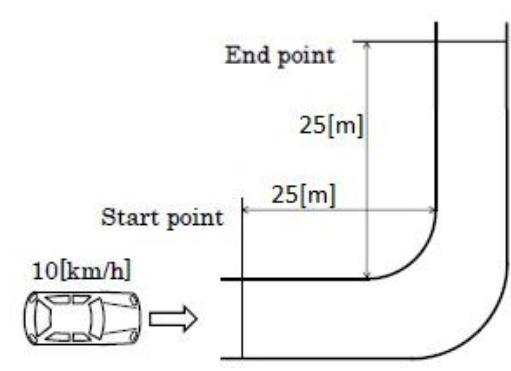

Fig. 3 The test course layout. Test subjects drove the test vehicle at $10 \mathrm{~km} / \mathrm{h}$.

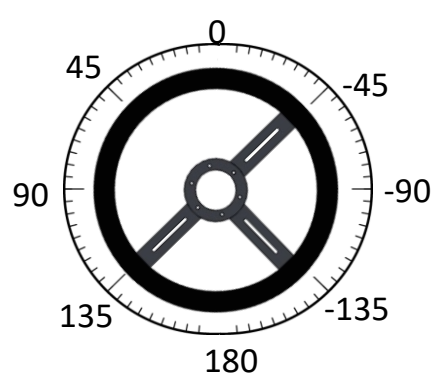

(a) Axes in Fig. 5.

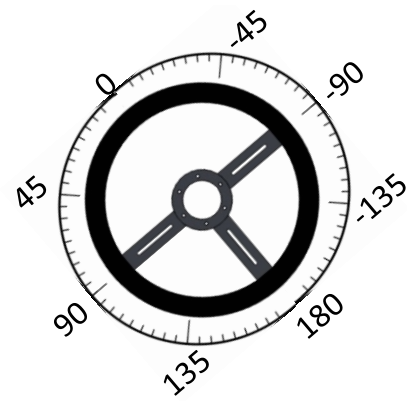

(b) Axes in Fig. 6.

Fig. 4 Visualization of the axes used in Fig. 5. Figure 6 (a) shows the axes used in Fig. 5 based on a fixed coordinate system. Figure 7 (b) shows the axes used in Fig. 6. The axes rotate with the steering wheel.

\subsection{Experimental result}

Figure 5 shows typical time-series data of the steering wheel angle, steering wheel grip position, and yaw angle for the driving of test subjects. The grip positions did not rotate with the steering wheel and were expressed as angles on absolute coordinates, taking the top-center of the wheel to be the origin 0 [deg] with a range of \pm 180 [deg], as shown in Fig. 4 (a). For both steering wheel and yaw angles, the counterclockwise direction was taken to be the positive direction. Discontinuous solid lines in Fig. 5 signify the removal of hands from the steering wheel, i.e., a grip change. The dotted lines indicate a neutral position. After the left turn and returning back to the straight line, the handgrip position returns to the dotted line when driving in a straight line. Figure 5 shows the time series data of the steering 
behavior. (a) shows the result for experienced driver. (b) shows the inexperienced driver.

Figure 5 (a) shows that the number of grip changes on the steering wheel was low (only four times) for the experienced driver. The experienced driver also demonstrated smooth steering, i.e., at the dotted line (yaw angle $\varphi=$ $90[\mathrm{deg}]$ and steering wheel angle $\theta=0[\mathrm{deg}]$ ), the steering wheel angle, yaw angle, and steering wheel grip position all converged to constant values immediately. On the contrary, the inexperienced driver demonstrated frequent grip changes, as shown in Fig. 5 (b); the steering wheel angle was 110[deg], and grip position of left and right hands were $\sim 45[\mathrm{deg}]$ and $200[\mathrm{deg}]$, respectively, at the straight-line-return dotted line $(\varphi=90[\mathrm{deg}])$, indicating that the steering has not been completed. Furthermore, the inexperienced driver required an additional grip change after the steering wheel angle returned to the neutral position because the grip positions were away from the neutral position. Furthermore, the inexperienced driver was meandering in other trial. The maximum steering wheel angle for the inexperienced driver was 526[deg], as opposed to the lower $415[\mathrm{deg}]$ of the experienced driver, and the steering holding condition was not exist. the steering holding condition refers to when the steering angle is held steady in a position other than the neutral position. A vehicle changes its direction with time through the integration of the yaw rate as long as there is a constant yaw rate matched to the curvature of the corner being run by simply holding a steady state of steering. This inexperienced driver did not understand this integral characteristic and continued to turn the steering wheel while driving around the corner. Therefore, when the inexperienced driver realized that the steering wheel needed to be returned to the central position, the steering angle was very large. The inexperienced driver rushed to return the steering wheel back to the central position but generated meandering.

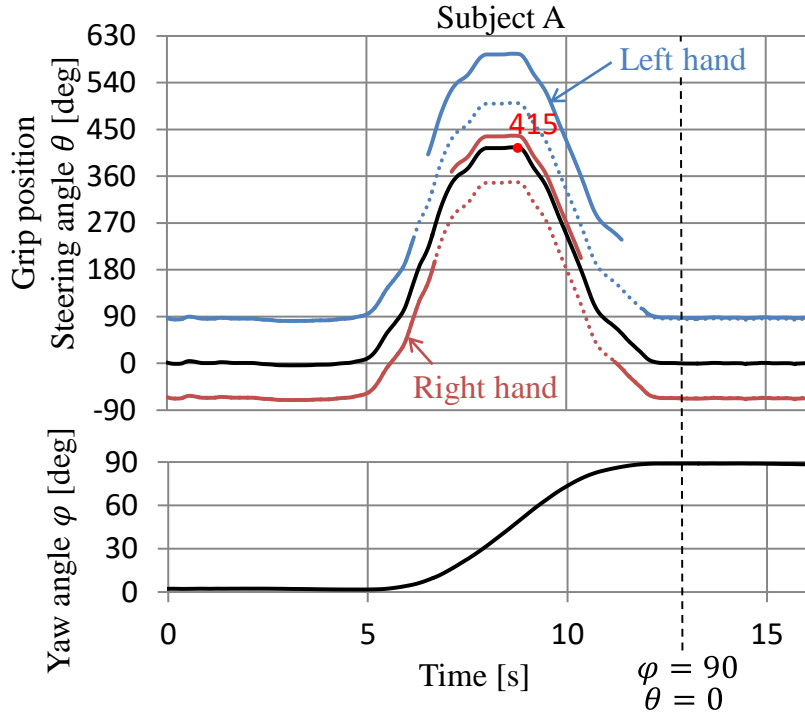

(a) Time-series data for the experienced driver.

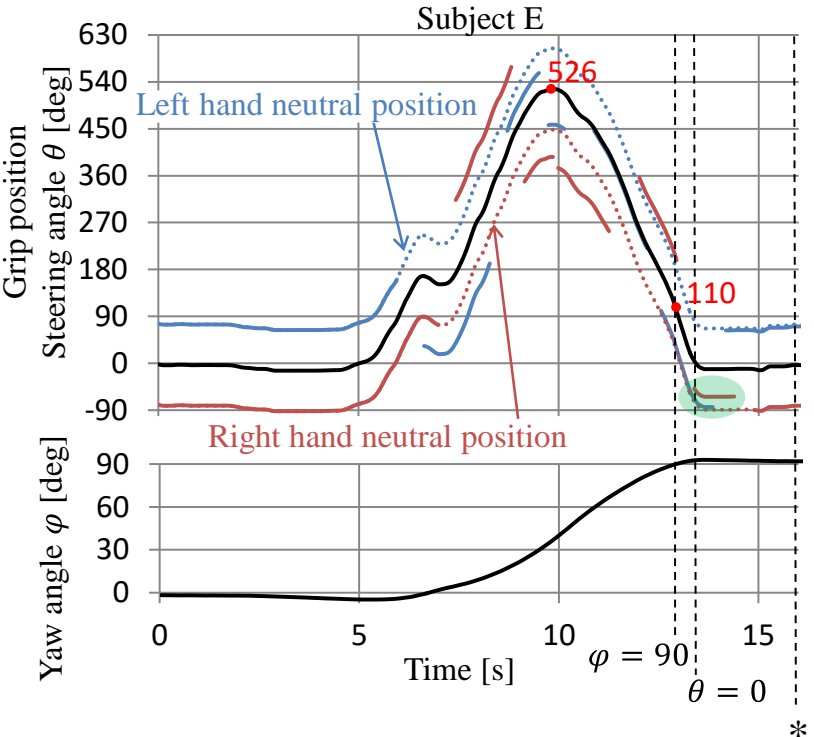

(b) Time-series data for the inexperienced driver.

Fig. 5 These figures show the time series of yaw angle, steering angle and steering grip position. In upper graph, the black line is the steering angle, the blue line is the steering grip position on the left hand, and the red line is the steering grip position on the right hand. The dotted line is the neutral position of the steering wheel. The driver grips this position during straight driving. In lower graph, the black line is the yaw angle. (a) is the result of experienced driver. In this case, the steering angle, the yaw angle and the steering grip position change converged at the same time. (b) shows the result of beginner driver. In contrast, it was not able to be converged these three terminal

Figure 6 shows the steering wheel grip position data overlaid three times from the same drivers as in Fig. 5. The coordinate system used in Fig. 6 differs from that used in Fig. 5 and rotates with the steering wheel, as shown in Fig. 4 (b). In other words, the coordinate system of Fig. 6 indicates the physical grip positions on the steering wheel. The horizontal solid line indicates that the grip is maintained at a fixed position until it is released. The line colors indicate the first, second, and third test runs in the order of decreasing color hue.

Looking at the results for the experienced driver, as shown in Fig. 6 (a), it can be seen that the variation in grip 
positions between runs was small and a similar steering action was performed in each run. Meanwhile, the results for the inexperienced driver in Fig. 6 (b) show large variations in the grip positions and the grip duration differs for each run. This showed a lack of reproducibility in the maneuvers of the inexperienced driver even on the same course.

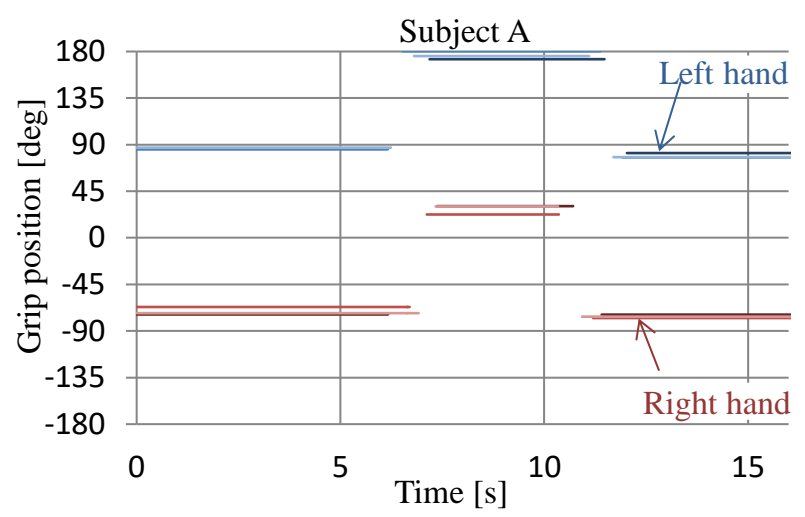

(a) Time-series data for the experienced driver.

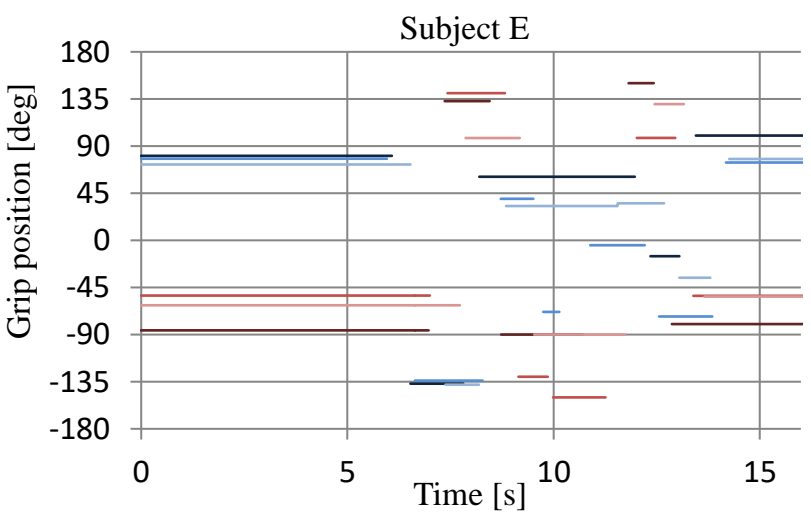

(b) Time-series data for the inexperienced driver.

Fig. 6 Time-series data of steering wheel grip positions for particular test subjects on the steering wheel coordinate system. Horizontal solid line shows a driver maintaining grip at the same position. Line color hue indicates the run order, where a darker hue indicates an earlier run. (a) shows the results for the experienced driver. This test subject used nearly identical handgrip positions in all three runs. (b) shows the results for the inexperienced driver. This test subject used varying handgrip positions for the three runs.

Figure 7 shows a plot of the steering wheel angle vs. the yaw angle for a particular test subject with all the runs overlaid. The black dots are plotted at the points where the steering wheel angle velocities become negative and signify the "return initiation positions."

The results for the experienced driver, as shown in Fig. 7 (a), show the waveform of the steering wheel angle is nearly symmetrical around the $45[\mathrm{deg}]$ yaw angle line. For the experienced driver, the variation in the maximum steering wheel angles is small (standard deviation $\sigma=15.9[\mathrm{deg}]$ ) and the yaw angles at return initiation are also consistent $(\sigma=7.1[\mathrm{deg}])$. This demonstrated a high degree of reproducibility in the steering action in each run of the experienced driver. The waveform also tends to be vertically flat around the maximum steering wheel angles. This indicates the steering holding condition; the driver decided the steering angle early on and held it while waiting for the yaw angle to change. This shows that this driver understood the aforementioned integral characteristic and was steering in a very relaxed state.

On the contrary, the results for the inexperienced driver in Fig. 7 (a) showed large variations in the maximum steering wheel angle (standard deviation $\sigma=34.3[\mathrm{deg}]$ ) and yaw angles at return initiation (standard deviation $\sigma=$ 14.0[deg]). This indicates that the inexperienced driver adopted a different course strategy and steering action for each trial. Moreover, the waveforms of the inexperienced driver are sharp near the maximum steering wheel angle, showing less the steering holding condition. This can be considered to indicate that the inexperienced driver is applying and returning the steering in a trial and error manner. 


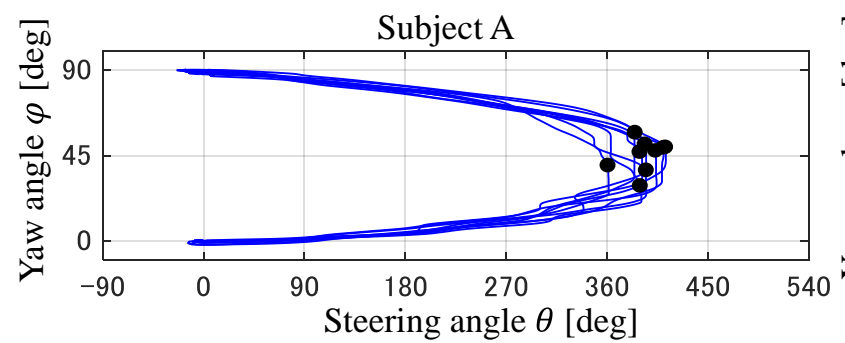

(a) Results for experienced driver.

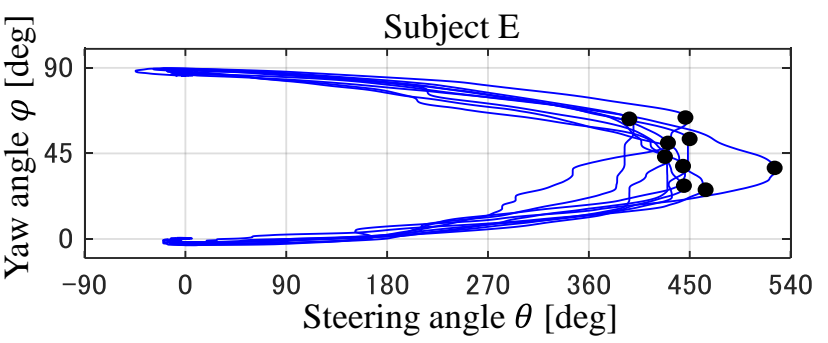

(b) Results for inexperienced driver.

Fig. 7 Relationship between the steering wheel angle and yaw angle. The results for all nine runs of test subjects $\mathrm{A}$ (experienced driver) and $\mathrm{E}$ (inexperienced driver) are shown. The waveform of test subject $\mathrm{A}$ is symmetric about the line yaw angle $\varphi=45[\mathrm{deg}]$. On the contrary, the waveform of test subject $E$ differs in each run.

The above experimental results demonstrated that when an inexperienced driver drove with a large curvature around a corner, a steering grip change was required, and there was little reproducibility in the steering action, regardless of experience on the course. The number of grip changes was also far higher for the inexperienced driver than the experienced driver. Furthermore, it was found that there were unstable elements, such as hesitation and meandering, in the steering of an inexperienced driver. In corners that require a grip change as the yaw angle reaches the target value, it is desirable that three conditions (1) straight-line vehicle motion, (2) steering wheel angle of $\theta=$ 0 [deg], and (3) neutral grip position, are all achieved simultaneously. Fulfilling such complex end conditions is difficult for an inexperienced driver, which leads to the aforementioned instability. On the contrary, experienced drivers demonstrated stable steering, and the aforementioned three conditions also converged smoothly after the corner.

It can be concluded that steering action that requires grip change relies heavily on ability learned through experience and practice, i.e., low-speed driving that involves frequent grip changes and steering is a non-intuitive task and adds to the potential driver workload.

Therefore, steering grip changes should be eliminated to simplify the three end conditions and create an improved steering system. In the next chapter, the development of a new steering system that would make grip changes unnecessary is discussed.

\section{Primary lab experiment}

To make a steering system without grip changes a reality, the steering ratio must be greatly decreased. However, simply applying a lower steering ratio to a conventional steering system would lead to losses in steering resolution and accuracy, making the car more difficult to drive. Therefore, a new steering system with improved control performance that does not lose its drivability, even with a low steering ratio, is proposed. First, a preliminary lab experiment was carried out to determine the specifications for the proposed steering system. In the preliminary lab experiment, the focus was placed on the direction of the driver's arm motions and grip form. Then, the effects of the arm motions and grip form on the control performance and required mental workload were considered through an experiment with human test subjects.

\subsection{Experimental specification}

Four different steering system specifications were prepared (Table 1). Figure 10 shows each of the steering systems. Specification (1) is a conventional steering system with a steering ratio of 20:1. Specification (2) has the same physical form as the conventional steering system, but with a steering ratio of 1:1; therefore, it does not require a grip change while steering. Specification (3) has the same steering column axis inclination angle as the conventional steering system, but uses a handlebar for the grip, with a steering ratio of 1:1. Specification (4) is similar to specification (3), but with a $90[\mathrm{deg}]$ steering column axis inclination angle and a back-and-forth driver arm motion. Specification (4) also uses a handlebar and has a steering ratio of 1:1. Grip diameter is the same in all specification. The reaction force to the front wheel angle is the same. This also applies to the following chapters 4 and 5 . 


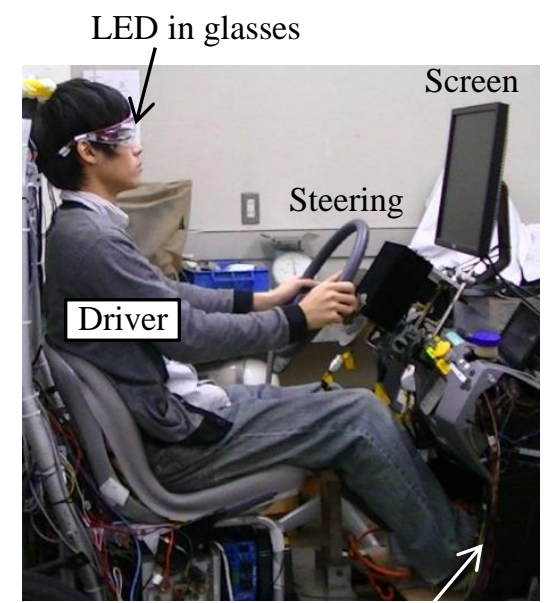

React by pushing the switch

Fig. 8 Experiment set-up. The test subjects wore glasses embedded with LEDs, and pressed the footswitch when they LEDs were illuminated.

Table 1 Steering system specifications.

\begin{tabular}{|c|c|c|c|}
\hline $\begin{array}{c}\text { Spec. } \\
\text { No. }\end{array}$ & Steering shape & $\begin{array}{c}\text { Axial angle } \\
{[\mathrm{deg}]}\end{array}$ & Gear ratio \\
\hline (1) & Wheel & 30 & $20: 1$ \\
\hline (2) & Wheel & 30 & $1: 1$ \\
\hline (3) & Bar & 30 & $1: 1$ \\
\hline (4) & Bar & 90 & $1: 1$ \\
\hline
\end{tabular}

\subsection{Experimental method}

To compare the maneuverability and driver mental margin during the steering maneuvers of each specification, an experiment with a dual-task paradigm was conducted. As shown in Fig. 8, the test subjects wore specially designed glasses and were seated in front of a screen. The screen displayed indicator bars, as shown in Fig. 9, and the test subjects were able to move the tracking bars side-to-side by moving the steering device. As the main task, the test subjects were asked to move the tracking bars to track the randomly moving target bars that corresponds to a front wheel angle. The cumulative error of the 'tracking' with respect to the target was used as the performance metric (Fig. $11)$.

The test subjects were also tasked with a sub-task in addition to the main task. The degree of driver mental margin was rated through the sub-task performance. The special glasses worn by the test subjects were fitted with LEDs that were randomly illuminated either blue or red. The illumination timing is shown in Fig. 12; the LEDs were randomly illuminated in either color for a duration of $1 \mathrm{~s}$ at an interval of 1 -s. The test subjects were asked to press a footswitch only when the LED was illuminated blue. In the sub-task, the reaction time taken to press the switch after the LED was illuminated was used as the performance metric for the driver mental margin. Ten male students who are average drivers in their twenties took part in the experiment. 


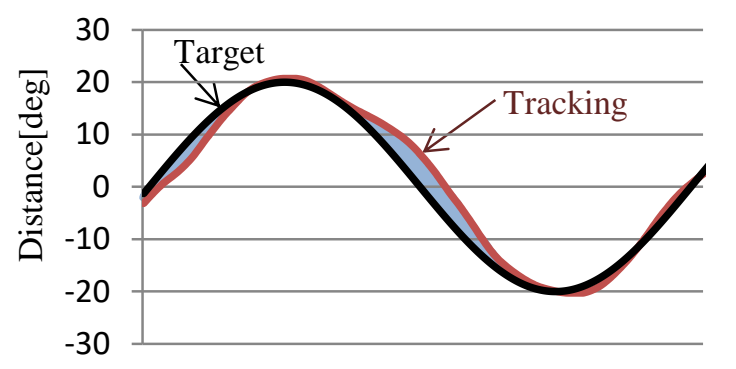

Fig. 11 Error between 'target' and 'tracking'. The cumulative sum of the error was used as the performance metric.

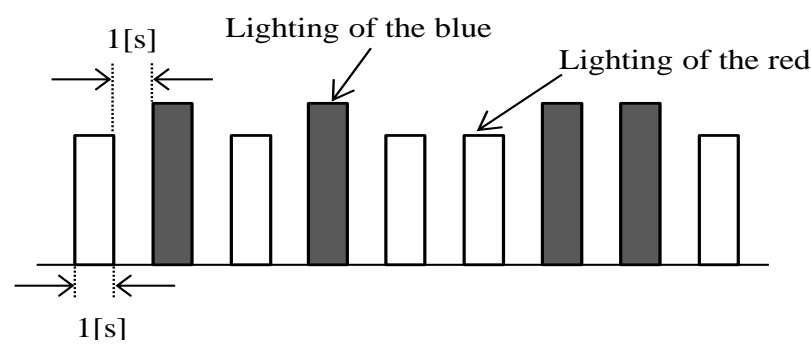

Fig. 12 Visualization of the timing of LED illuminations. The LEDs were randomly illuminated blue or red.

\subsection{Experimental result}

Figure 13 shows the relation between the tracking errors and reaction time taken to press the footswitch after the illumination of the LED. First, the effect of grip change or lack thereof was considered. When specification (1) ( $\mathbf{\Delta}$ ), a conventional steering system, was used, both the errors and reaction times were large. When specification (2) ( $\square$ ), with a steering ratio 1:1 that does not require a grip change was used, both the errors and reaction times were reduced. From this, we can see that grip change adversely affects the maneuverability while simultaneously increasing the mental workload.

Then, results from cases with different grip shapes but identical steering ratios were compared. Comparing the result of specification (2) ( $\square$ ) with a steering wheel to that of specification (3) (x) with a handlebar, both with a steering ratio of $1: 1$, it can be seen that specification (3) ( $\mathbf{x}$ ) had both smaller errors and reaction times. From this, it can be seen that steering with a handlebar reduces the mental workload and is easier even with a low steering ratio. Furthermore, the results from cases with equal steering ratios and grip shapes but different driver arm motions were compared. It can be seen that specification (4) ( $\bigcirc$ ), which involves the back-and-forth motion of the driver arms, reduced the errors and improved the maneuverability compared to specification (3) (x), which involves the up-and-down motion of the driver arms similar to a conventional steering system.

From these experimental results, it was found that improving the maneuverability and reducing the mental workload of the driver would be possible if the need for grip change could be eliminated. It was also observed that using a steering system with a handlebar that involves the back-and-forth motion of the driver's arms allowed for easy and intuitive steering even with a low steering ratio.

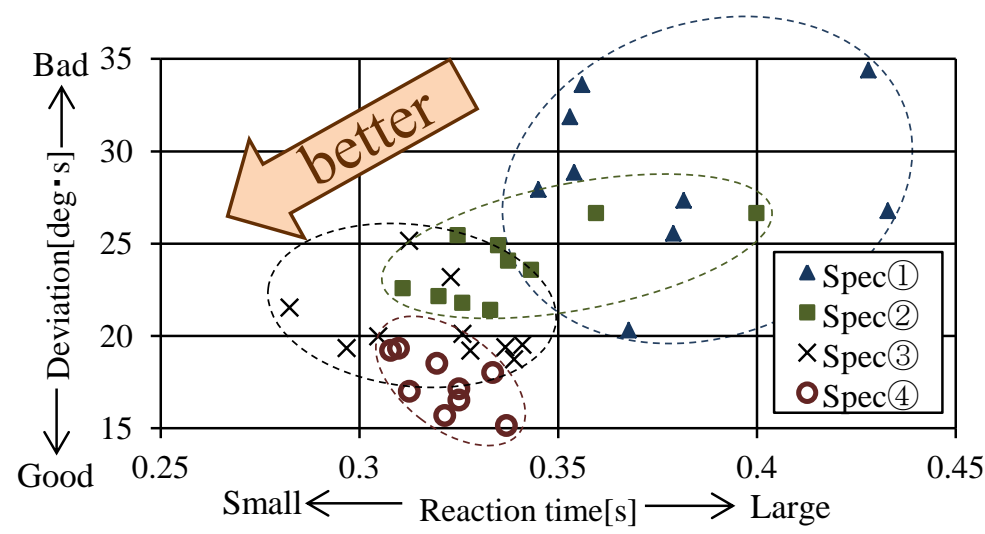

Fig. 13 This figure shows the relationship between the tracking errors and the reaction time taken to press the footswitch after LED illumination. $\boldsymbol{\Delta}$ denotes specification (1), $\mathbf{a}$ denotes specification (2), $\times$ denotes specification (3), and $\circ$ denotes specification (4). By eliminating grip changes, errors were reduced and reaction times were shortened. Also, the control performance was improved further when the steering system grip was changed to a handlebar with a back-and-forth driver arm motion. 


\section{Driving simulator experiment \\ 4.1 Experiment overview}

In Chapter 3, the preliminary lab experiment demonstrated that a steering system specification using a handlebar for the steering grip and a back-and-forth motion for the driver's arms is advantageous for improving the maneuverability and reducing driver mental workload. In this chapter, the feasibility of the real-life application of the four specifications is verified. The experiment was conducted using a driving simulator setup, as shown in Fig. 14. This device calculates the vehicle motion based on the driver steering wheel angle input and gives the driver visual feedback and steering reactive feedback torque. A DSP controller (AD5435) from A\&D Company Ltd was used for various types of signal processing and vehicle motion calculations in this experiment. For vehicle animation, the Carsim software from Virtual Mechanics was used.

The test subjects were the same ten male students who are average drivers in their twenties as in the preliminary experiment. Lane changes were performed five times while driving at a constant speed of $20 \mathrm{~km} / \mathrm{h}$. All test subjects were driver's license holders. Figure 15 shows the course layout. The simulator automatically adjusted the speed, and thus the test subjects were only required to provide steering input. The test subjects were tasked with driving while following the target course drawn on the test course. The cumulative error between the target course and the car position and total yaw angle $\varphi^{*}$ was used as the performance metric.

Equation (1) shows the calculation method for the total yaw angle $\varphi^{*}$. The total yaw angle $\varphi^{*}$ is the integral of absolute value of yaw rate. If a driver applies corrective or unnecessary steering or causes erratic vehicle motion during the test runs, the total yaw angle $\varphi^{*}$ takes a large value and indicates poor drivability. The total yaw angle $\varphi^{*}$ does not accumulate in straight-line sections with zero yaw angle; hence it did not matter if different test subjects drove through straight-line sections with various lateral offsets.

$$
\begin{array}{r}
\varphi^{*}=\int|\gamma| d t \\
\gamma: \text { Yaw rate }
\end{array}
$$

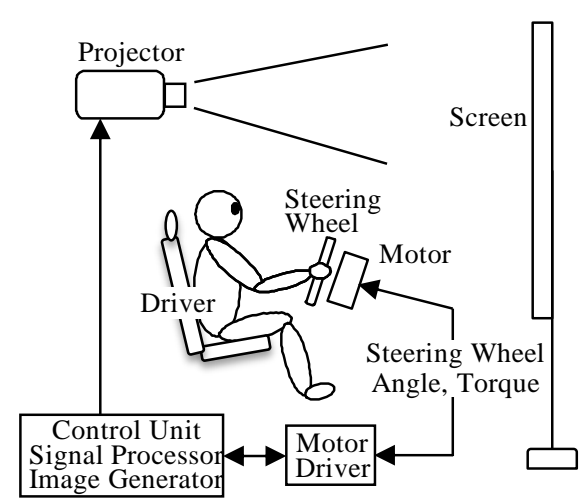

Fig. 14 Driving simulator setup. The test subjects drove the test car at a constant $20 \mathrm{~km} / \mathrm{h}$. The test car speed was maintained automatically and the test subjects only provided the steering input.

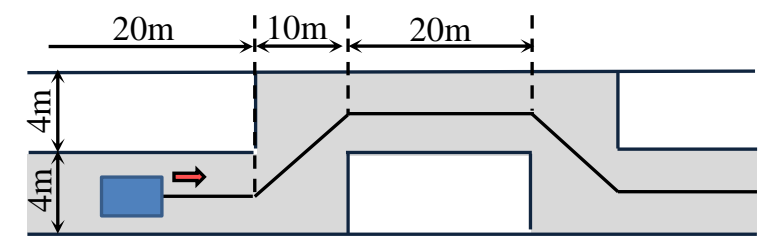

Fig. 15 Test course layout driven through in the driving simulator. The test subjects performed lane changes while following a target course.

\subsection{Experimental result}

Figure 17 shows a representative time-series data of the front wheel angle for a particular test subject. The front wheel angle was used instead of the steering wheel angle because the steering ratios differed according to the specification used. When driven using the conventional steering system of specification (1) ( $\boldsymbol{\Delta}$ ), the delay in steering input was noticeable and took a long time to stabilize. On the contrary, when specification (2) (匹) with a 1:1 steering ratio was used, the initial rate of the steering was fast but the peak front wheel angle was very large and frequent corrective steering was observed. It is assumed that while a low steering ratio allowed the driver to react quickly, fine steering adjustments became difficult due to reduced steering resolution. When steering system specification (3) ( $x$ ) 
with a handlebar grip was used, the maximum front wheel angle was kept low, and there was good stabilization and little delay in steering. When specification (4) (O) with back-and-forth arm motion during steering was used, the driver was able to steer more smoothly with less steering input compared to specification (3) ( $\times$ ). This trend was consistent across all test subjects.

Figure 18 shows the relation between the cumulative error between the car position and the target course and total yaw angle $\varphi^{*}$. When specification (1) ( $\Delta$ ) was used, the positional error against the target was high. This error was reduced when specification (2) ( $\mathbf{\square}$ ), which has a lower steering ratio than specification (1) ( $\boldsymbol{\Delta}$ ), was used. However, the total yaw angle $\varphi^{*}$ increased and corrective steering inputs were required when specification (2) (匹) was used. On the other hand, when specification (3) ( $\mathbf{x}$ ), with a handlebar handgrip was used, the error and total yaw angle $\varphi^{*}$ were small. Furthermore, when specification (4) ( $\bigcirc$ ), with a back-and-forth driver arm motion, was used, the variations between test subjects were reduced; both the error and total yaw angle were small, and the test subjects were able to track the target using smooth steering inputs.

Thus, specification(4) $(\bigcirc)$, which allowed the drivers to drive smoothly even with a low steering ratio of $1: 1$, was selected as the proposed steering system to be developed further in this research.

Now, while the experiment was conducted at low-speed driving conditions, we assume that it is possible to apply the system to high-speed driving by controlling the steering ratio adapting to the car velocity. Furthermore, although this experiment was primarily concerned with the control performance during short driving sessions, the system could reduce driver workload in extended driving sessions as well.

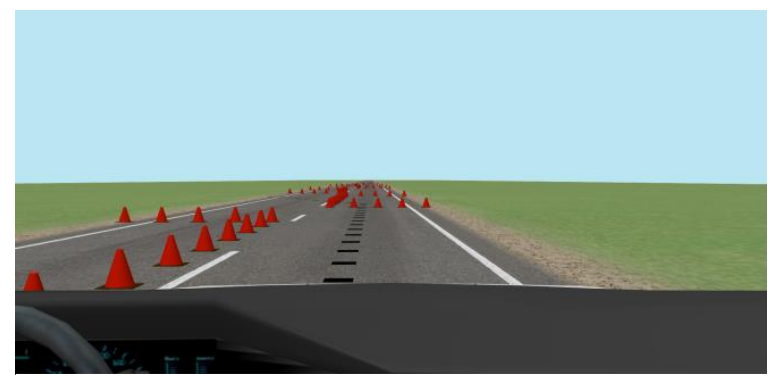

Fig. 16 Driver view in the driving simulator. The dotted line in the middle of the lane is the target course.

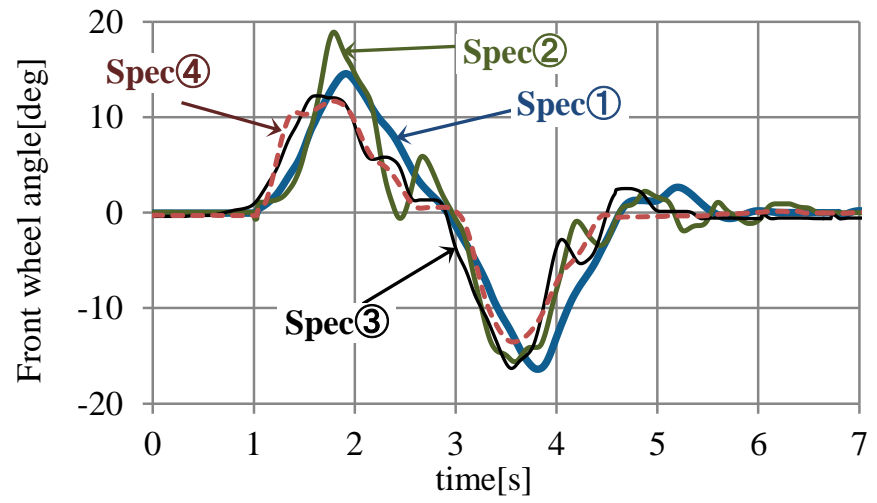

Fig. 17 Time-series data of the front wheel angle. The blue solid line denotes specification(1); the green solid line denotes specification(2); the black solid line denotes specification(3), and the red dotted line denotes specification (4). When using a conventional steering system, specification (1), the delay in steering and poor convergence is noticeable. On the other hand, specification (4) reduced steering delay and converged quickly. 


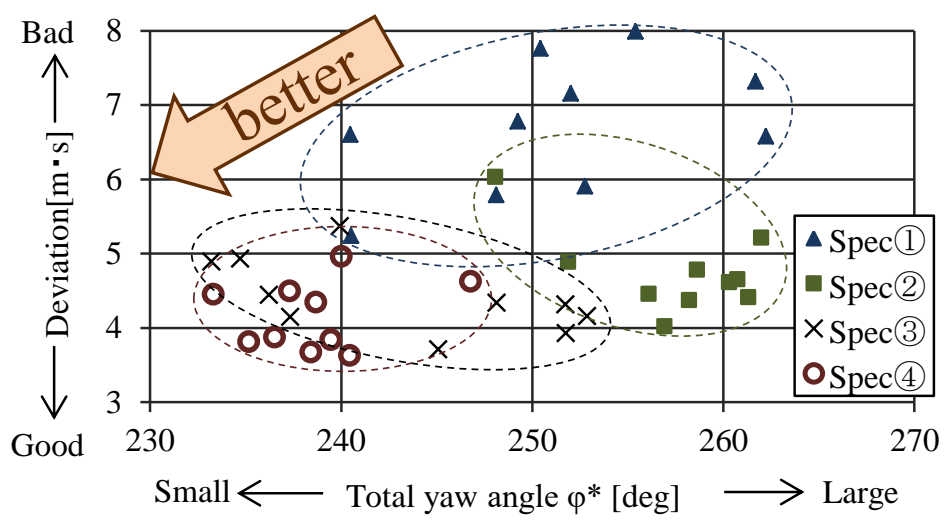

Fig. 18 Relationship between the cumulative error between the car position and the target course and total yaw angle $\varphi^{*}$. $\Delta$ denotes specification (1), - denotes specification (2), $\times$ denotes specification (3), and $\bigcirc$ denotes specification (4). When using specification (1) ( $\mathbf{\Delta})$, both the cumulative errors and total yaw angles $\varphi^{*}$ were high. In contrast, both the errors and total yaw angle $\varphi^{*}$ were low when specification(4) $(\bigcirc)$ was used.

\section{Variation in driver characteristics due to steering system shape 5.1 Forward first-order predictive model}

Figure 19 is a block diagram of the forward preview first-order predictive model. The driver model steers the vehicle to minimize the error $e_{y}$ between the vehicle position at the preview point $y_{p}$ and the corresponding target point $y_{p}{ }^{*}$.

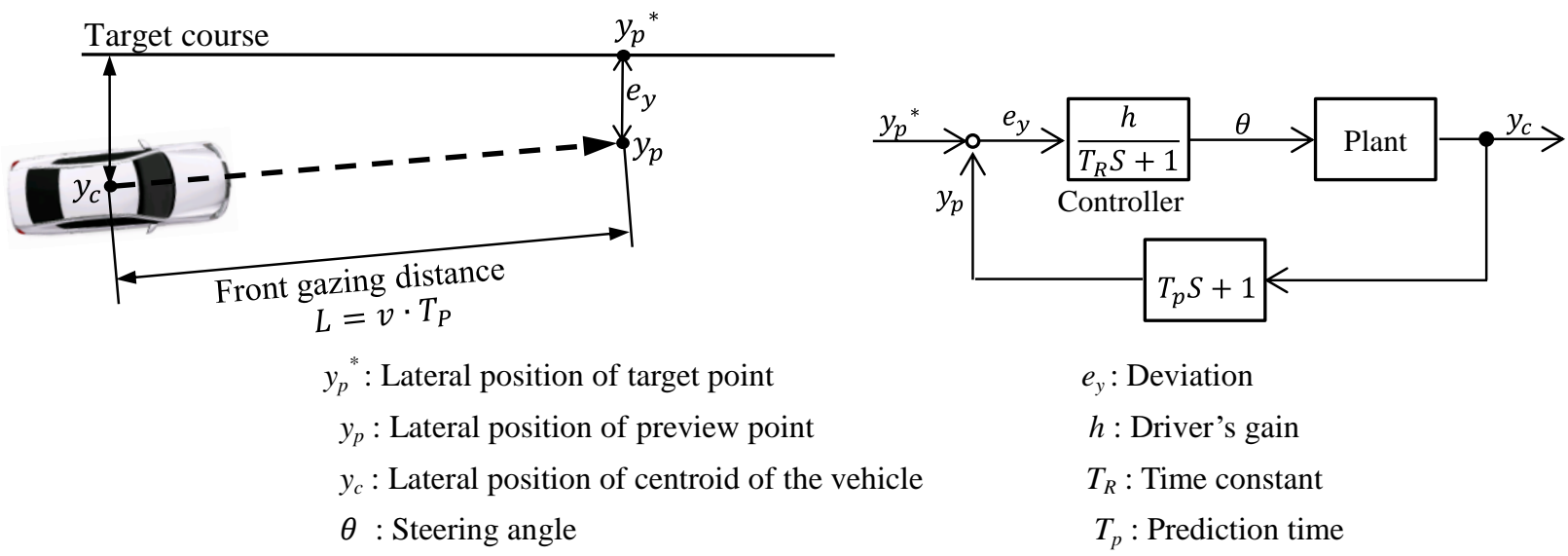

Fig. 19 Forward First-Order Predictive Model and its block diagram. The driver model steers to minimize the error $e_{y}$ between the vehicle position at preview point $y_{p}$ and corresponding target point $y_{p}{ }^{*}$.

\subsection{Experimental method}

An experiment using a driving simulator to investigate the effect of the steering system shape on the driver characteristics was conducted. We identified drivers transfer function (Fig.19) by ARX model (Suzuki et al., 2014). Then the input was the error $e_{y}$ between the vehicle position at the preview point $y_{p}$ and the corresponding target point $y_{p}{ }^{*}$, and the output was the driver steering operation. Three steering system specifications were prepared for the experiment: a conventional steering system with a steering wheel (wheel (20:1)), a similar specification but with a low 1:1 steering ratio to eliminate grip change (wheel (1:1)), and the proposed steering system with handlebar grip as explained in Chapter 4 (bar (1:1)). Except for the steering system shape and steering ratio, the vehicle dimension, and steering grip diameter, among others, were standardized for all specifications. The reaction force to the front wheel angle is the same. The car speed during test runs was set at $20 \mathrm{~km} / \mathrm{h}$, and the simulator automatically made speed adjustments. The steering ratios used were 20:1 for wheel (20:1) and 1:1 for wheel (1:1) and bar (1:1). The test subjects were eight male students who are average drivers in their twenties. 
Table 2 Steering system specifications.

\begin{tabular}{|c|c|c|}
\hline Spec name & Shape & Gear ratio \\
\hline Wheel(20:1) & Wheel & $20: 1$ \\
\hline Wheel(1:1) & $\downarrow$ & $1: 1$ \\
\hline Bar(1:1) & Bar & $\downarrow$ \\
\hline
\end{tabular}

Fig. 20 Test course layout. The target course (lateral position $=0$ $\mathrm{m})$ is displayed on the left lane. The test subjects initially drive along the target course, then the vehicle receives an external disturbance which instantaneously offsets the vehicle position into the right lane (lateral position= $-3.5 \mathrm{~m}$ ). The test subjects then steer to return to the target course. The recovery maneuver was measured to determine driver characteristics (driver gain $h$, time constant $T_{R}$ ). The control performance was also evaluated from the recovery maneuver.

The target course (lateral position $=0 \mathrm{~m}$ ) was displayed in the middle of the left lane in a two-lane road, as shown in Fig. 20. The test subject initially drives along the target course and then receives an external disturbance that instantaneously offsets the vehicle into the right lane (lateral position $=-3.5 \mathrm{~m}$ ) without steering reaction. The test subject then steers the vehicle back to the target course. The recovery maneuver was measured to determine the driver characteristics (driver gain $h$, time constant $T_{R}$ ). Also, while the steering wheel angle is normally used as the driver input for driver characterization, this experiment used the front wheel angle as the driver input to avoid steering ratio effects.

In addition, the total front wheel angle $\delta^{*}$ during the recovery maneuver was calculated by Eq.(2) to be used as a metric for control performance. The total front wheel angle $\delta^{*}$ is the integral of absolute value of front wheel angular velocity. This index shows a large value if the driver generates corrective or erratic steering, and can express the driving difficulty which cannot be fully explained by a transfer function.

$$
\begin{aligned}
& \delta^{*}=\int|\dot{\delta}| d t \\
& \dot{\delta} \text { : Front wheel angular velocity }
\end{aligned}
$$

\subsection{Experimental result}

Typical time-series data of front wheel angle for particular test subjects are shown in Fig. 21(a). When the results of driving with wheel $(20: 1)$ are compared against wheel (1:1), it can be seen that, when wheel(1:1) is used, the initial rise of the front wheel angle is sharp, and there is a high front wheel angular velocity. The angular velocity increasing with a lower steering ratio using a steering wheel is a typical response. The maximum front wheel angle was also large and small corrective steering inputs were visible. Hence, it can be seen that if the steering ratio is lowered while keeping a steering wheel, driving would be more difficult despite the need for grip change being eliminated.

On the contrary, when bar (1:1) with handlebar grip was used, the front wheel angle had a smaller amplitude with smoother steering, and it could be seen that driving is easy even with a low steering ratio.

The relation between the total front wheel angle $\delta^{*}$ and steering gain $h$ for each of the test subjects, along with the determination of the time constant $T_{R}$, is shown in Fig. 21(b). When wheel (20:1) ( $\Delta$ ) or wheel (1:1) ( $\square$ ) were used, both $\delta^{*}$ and $h$ were distributed consistently in close range for each test subject. However, when wheel (1:1) ( $\square$ ) was used, the points greatly varied between the test subjects, and it could be seen that the test subjects were not driving similarly. The time constant $T_{R}$ also becomes lower with a lower steering ratio, indicating the loss of mental margin in carrying out the steering maneuver. When wheel (20:1) ( $\Delta$ ) was used, the value of $T_{R}$ was much greater when compared with the other specifications. This was because the steering velocity could not be sufficiently generated.

Meanwhile, when bar (1:1) ( $\circ$ ) was used, the steering gain $h$ value obtained was lower than those obtained by the other two specifications. This indicates that the driver steering gain is small when reacting to the positional error 
against the target course at the preview point and that the driver is steering in a relaxed state. Also, when bar (1:1) ( 0 ) was used, the value of total front wheel angle $\delta^{*}$ was smaller than those of the other two specifications, showing that bar (1:1) (०) is a steering system specification that is easy to drive. In terms of the time constant $T_{R}$, the value was larger for bar $(1: 1)(\circ)$ than wheel $(1: 1)(\varpi)$. This shows that the proposed steering system with a handlebar grip allows for a more relaxed steering at the same steering ratio.

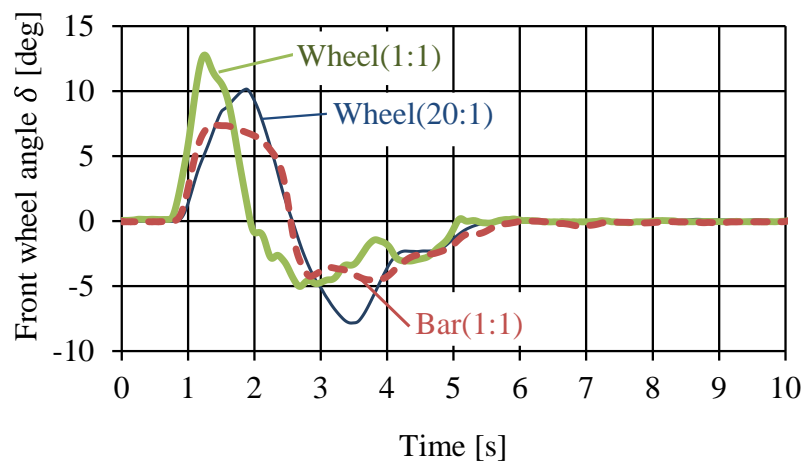

(a) Time-series data of the front wheel angle.

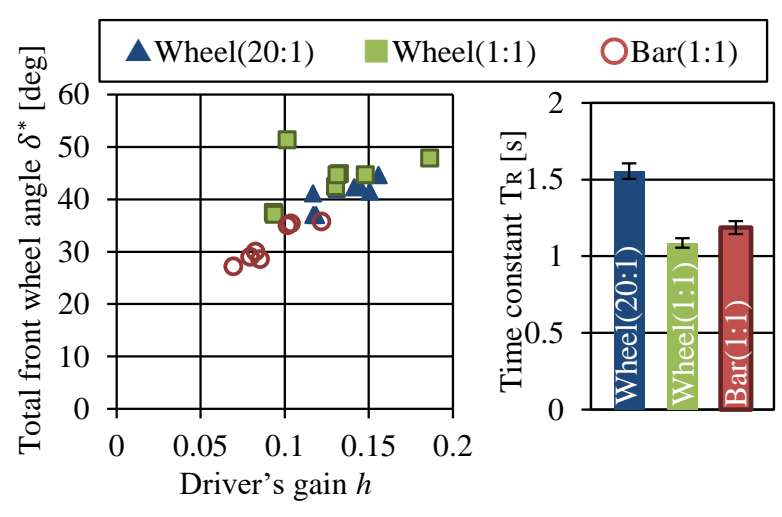

(b) Driver characteristics.

Fig. 21 Results of the driving simulator experiment. (a) shows the typical time-series data of the front wheel angle. The blue solid line, green solid line, and red dotted line indicate wheel (20:1), wheel (1:1), and bar (1:1), respectively. When wheel (1:1) was used, the value of the maximum front wheel angle was largest among the specifications and many corrective steering actions were performed. In contrast, when bar (1:1) was used, the value of the maximum front wheel angle was the smallest of all specifications and the waveform was smooth. (b) shows the relationship between the driver gain $h$, total front wheel angle $\delta^{*}$, and time constant $T_{R}$. The driver gain $h$ and total front wheel angle $\delta^{*}$ were smallest for bar (1:1). The time constant $T_{R}$ was larger for bar (1:1) than Wheel (1:1). This shows that bar $(1: 1)$ is easier to drive than Wheel (1:1) when using a low steering ratio. Therefore, $\operatorname{Bar}(1: 1)$ is maneuverable even if it has low steering gear ratio.

In this way, we found that when a steering system with a steering wheel is used with a low steering ratio, the steering becomes unstable and difficult to drive; however, using the proposed steering system with a handlebar grip, it would be possible to achieve stable steering even with a low steering ratio. We also found that even with the same vehicle dimension and driver, the driver characteristics can vary depending on the shape of the steering system. Possible reasons include changes in the driver mental steering model and variations caused by the use of different muscle groups when using differently shaped steering systems. However, clarifying the specific details shall remain a future task.

\section{Real car experiment using the proposed steering system \\ 6.1 Test car}

A test car with the proposed steering system was manufactured to evaluate the compatibility of the system and real car. Figure 22 shows the driver's seat views for the test car. The vehicle was based on a normal left hand drive passenger car. A steer-by-wire conversion was carried out on the vehicle, and then the proposed steering system with a handlebar grip (Fig. 22(a)) was installed on the right seat side (It is also possible to replace the grip of the steering system with a conventional steering wheel (Fig. 22(b)) on the right seat side.). Figure 23 shows the steer-by-wire system schematic. The steering wheel angle was measured through an encoder fitted to the rotational axis of the steering system on the right seat side. The measured steering wheel angle was then relayed to the steering system on the left seat side through a newly installed electric motor that applied the driving torque on the steering column. An electric motor was also fitted on the right seat side steering system to generated steering reaction torque for the driver. Furthermore, the left seat side still has the original conventional steering system installed, and the steering inputs from this steering system are prioritized in an emergency. The throttle and brake pedals were also converted to throttle-by-wire and brake-by-wire systems, respectively, and identical systems were fitted to both the left and right seat 
sides. In an emergency, pedal inputs from the left seat side are also prioritized.

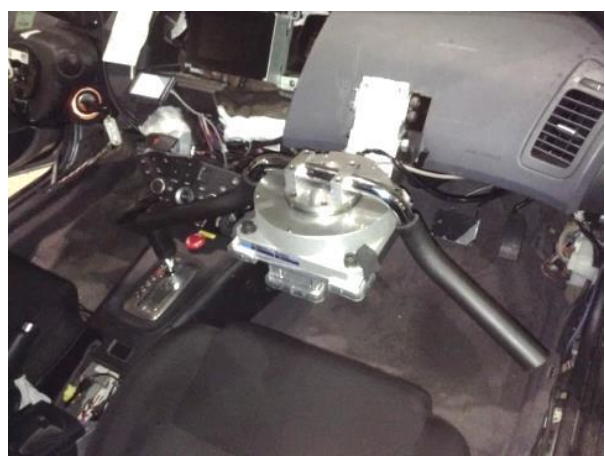

(a) Proposed steering system.

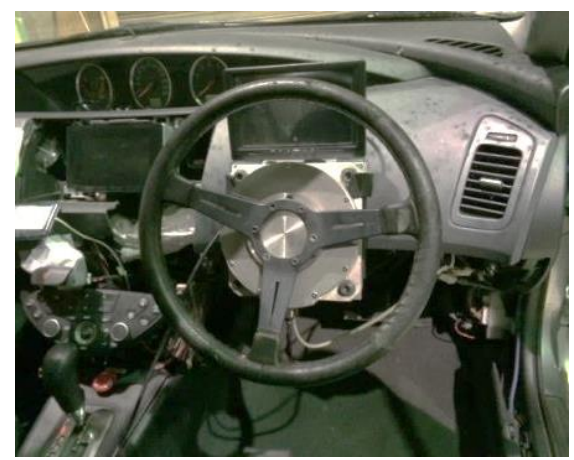

(b) Conventional steering system.

Fig. 22 Driver's seat configurations for the test car. (a) shows the proposed steering system.

(b) shows a conventionally shaped steering system. The driver is given reaction torque according to the steering wheel angle by an electric motor.

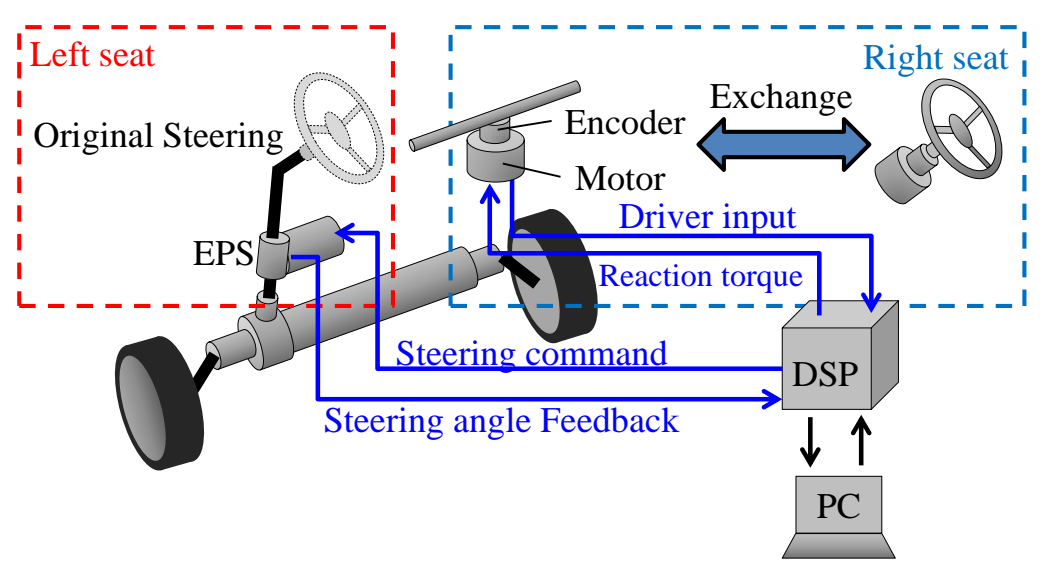

Fig. 23 Schematic of the steer-by-wire system on the test car. The driver steering wheel angle input is measured by the encoder fitted to the rotation axis of the steering system on the right side of the seat; it is then applied through an electric motor fitted to the steering column on the left side of the seat.

\subsection{Experimental method}

The possible steering system configurations for the test car were the proposed steering system with a handlebar grip, as shown in Fig. 22 (a), and the steering system fitted with a conventional-type steering wheel, as shown in Fig. 22 (b). For the steering ratio, the proposed steering system used 1:1 and the conventional-type steering system used 15:1, which was the steering ratio of the base car. The spring constant and viscous damping and friction coefficients for the steering reaction torque were decided to reproduce the real vehicle that (Table 3). These parameters were decided based on the conventional steering system, and we set 15 times them for the proposal system. The test subjects selected were four people with no previous car driving experience (non-experienced drivers) and four people who drive a car by commuting or going to school in daily life and over 5 years have passed since got driver's license for experienced drivers (experienced drivers). The skills acquired by training etc. are not instinctive motions of human beings, so it is assumed that there is not a little mental workload. Therefore, we verified intuitive operability from comparing the non-experienced drivers and the experienced drivers. Each of the test subject groups was further divided into two groups and the steering system specifications that were driven first in each subgroup were switched to eliminate the order effect. No practice runs were allowed to assess the intuitiveness of the steering systems. Each driver ran each steering specification four times. The drivers operated only steering, and the vehicle speed was adjusted automatically to $10 \mathrm{~km} / \mathrm{h}$. The test course consisted of a perpendicular left turn as shown in Fig. 24. 
The total excess yaw angle $\varphi_{\text {ext }}$, as defined in Eq. (3), was used as a performance metric. This metric includes a quantity of meandering and extra steering until reaching the target yaw angle ( $\cong 90[\mathrm{deg}]$ in this experiment), and a large value signifies a large amount of unnecessary motion.

Sufficient considerations were made for safety; ample space was provided on the out of test course. Also, a staff member seated in the left seat constantly monitored the safety and was able to apply the brakes or take over the steering in a dangerous situation.

Table 3 Steering system specifications for the experiment.

\begin{tabular}{|l|c|c|}
\hline & Proposal system & Conventional system \\
\hline Gear ratio & $1: 1$ & $15: 1$ \\
\hline Stiffness[Nm/deg] & 0.12 & 0.008 \\
\hline Viscosity[Nms/deg] & 0.09 & 0.006 \\
\hline Friction[Nm] & 0.12 & 0.008 \\
\hline
\end{tabular}

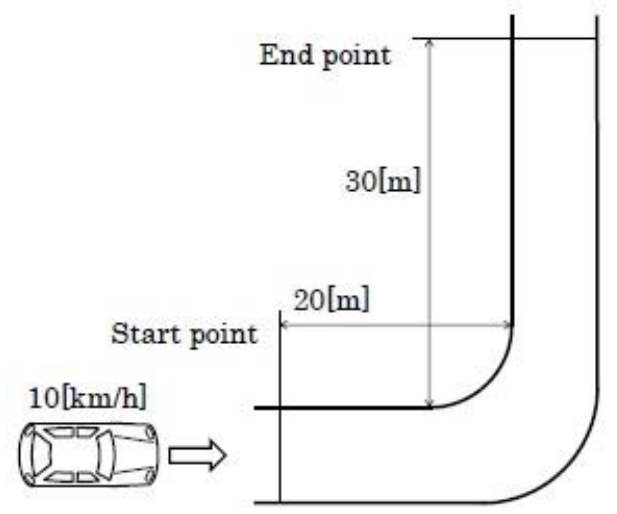

Fig. 24 Test course layout. Test subjects drove through this course at $10 \mathrm{~km} / \mathrm{h}$ in the test car.

$$
\varphi_{\text {ext }}=\int|\gamma| d t-\int \gamma d t
$$

$\gamma$ : Yaw rate

\subsection{Experimental result}

A time-series data of the front wheel angle for a particular non-experienced driver is shown in Fig. 25. The results (a), from when the conventional-style steering wheel was used for driving, show that there was a large scatter between the test runs, times taken to reach the maximum front wheel angle, and the values of the maximum front wheel angle. There were also multiple instances of corrective steering. Furthermore, this test subject was unable to change the steering angle smoothly to $0[\mathrm{deg}]$ when transitioning from the corner to the straight-line and meandered on a number of occasions. The driver commented, "The car did not turn as much as I thought when I turned the steering wheel" and "When I returned to the straight line, I could not find the center position of the steering wheel, so I meandered." This implies that the relation between the steering wheel angle and the front wheel angle had not been grasped by the driver.

On the contrary, in the case of using proposed steering system (b), no significant variations were observed between each trial, and corrective steering and meandering were hardly seen. Also, while the maximum front wheel angle when driving with the conventional-style steering system averaged 21.1[deg] $(\sigma=4.28[\mathrm{deg}])$, this was decreased to $17.0[\mathrm{deg}](\sigma=0.73[\mathrm{deg}])$ for the proposed steering system. The driver commented, "I felt that I could move the vehicle the way I wanted to" and "I was able to concentrate on the driving because I was not so busy turning the steering wheel." It was observed that the driver was able to drive intuitively and that the proportion of attentional resources allocated to steering had been reduced. Furthermore, one of the test subjects have never ridden a bicycle, which also has a handlebar type steering system, but had no problem with the steering. Hence, the result does not appear to depend on having experience with similar steering systems. 


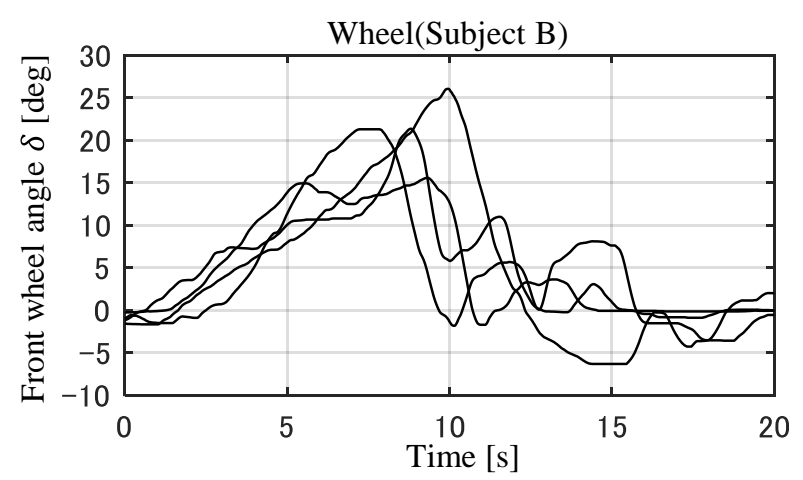

(a) Conventional-style steering system.

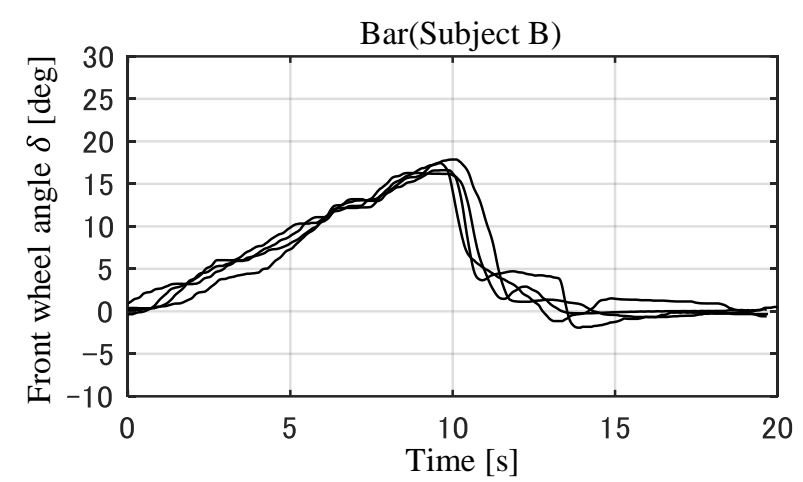

(b) Proposed steering system.

Fig. 25 These plots show time-series data of front wheel angles for a driver with no car driving experience. (a) shows the result for the conventional-style steering system with a steering wheel. In this case, the values of the maximum front wheel angle were different in each test run. There were also multiple instances of corrective steering. (b) shows the result for the proposed steering system with a handlebar. In contrast with (a), this test subject was able to steer with high reproducibility in all test runs.

Figure 26 shows the experimental results of an experienced driver. Comparing the results from driving with the conventional-style steering system (a) and the proposed steering system (b), it can be seen that the driver was able to drive without noticeable variations in the waveforms between runs or erratic steering maneuvers. While the maximum front wheel angle when driving with the conventional-style steering system averaged $24.4[\mathrm{deg}](\sigma=1.92[\mathrm{deg}])$, this was decreased to $17.9[\mathrm{deg}]$ (standard deviation $\sigma=0.58[\mathrm{deg}]$ ) for the proposed steering system. Furthermore, when the test subject drove using the proposed steering system, the variation in the waveform around the maximum front wheel angle is smaller, showing that the steering was performed in a more relaxed manner. This trend was consistent in the other test subjects.

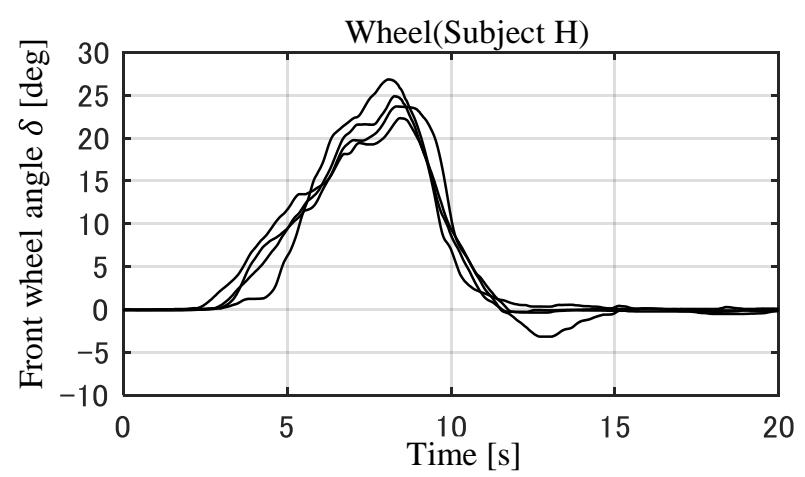

(a) Conventional-style steering system.

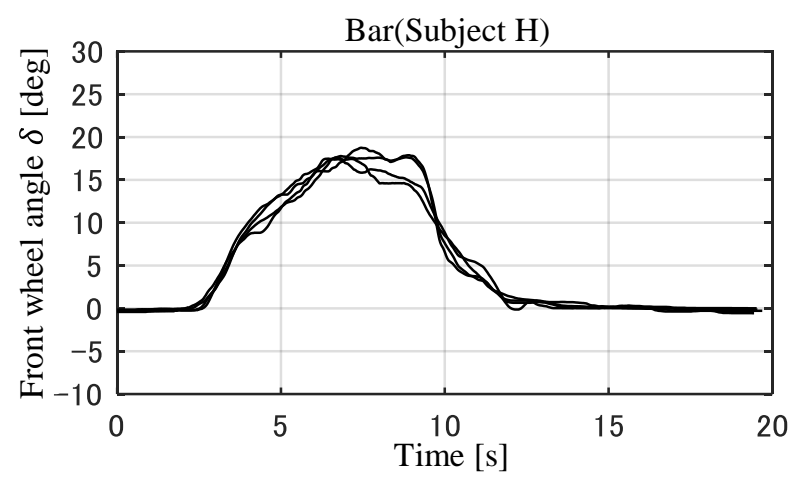

(b) Proposed steering system.

Fig. 26 These plots show time-series data of front wheel angles for an experienced driver. This test subject was able to drive smoothly with either specification. However, the maximum front wheel angle was larger for the conventional-style steering system than the proposed steering system.

Figure 27 shows the total excess yaw angle $\varphi_{\text {ext }}$ calculated from each experiment data. Results obtained for the non-experienced driver (a) show that the value of the total excess yaw angle was smaller for the proposed steering system than the conventional-type steering system, and unnecessary movements were greatly reduced. In particular, test subject D repeatedly meandered after turning around the corner when using the conventional-type steering system, resulting in a very high value of total excess yaw angle $\varphi_{\text {ext }}$. However, when the same test subject used the proposed steering system, he was able to transition smoothly into the straight-line section, and the value of the total excess yaw angle $\varphi_{\text {ext }}$ was low. In this way, it was observed that the proposed steering system allows even a non-experienced driver to steer smoothly and greatly suppresses instability and meandering in comparison with the conventional-style 
steering system. On the contrary, there were no large differences in total excess yaw angle $\varphi_{\text {ext }}$ for the experienced drivers between two steering system specifications. From this, it could be seen that using the proposed steering system presents no problems to drivers who are accustomed to conventional steering wheels.

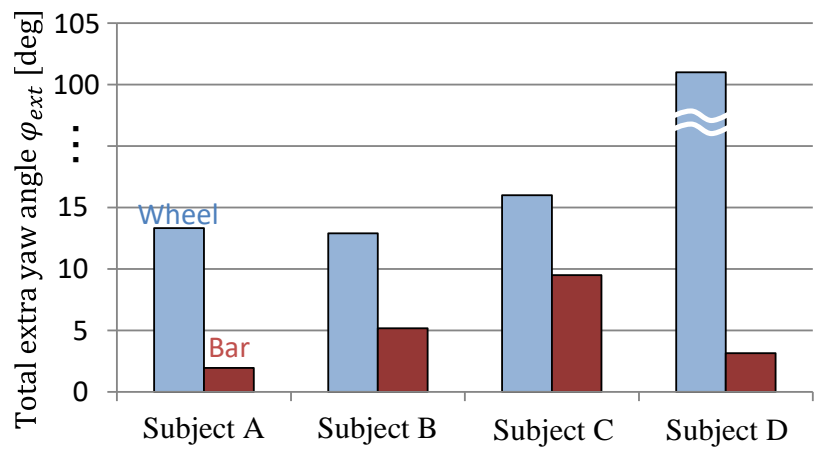

(a) Results for non-experienced drivers.

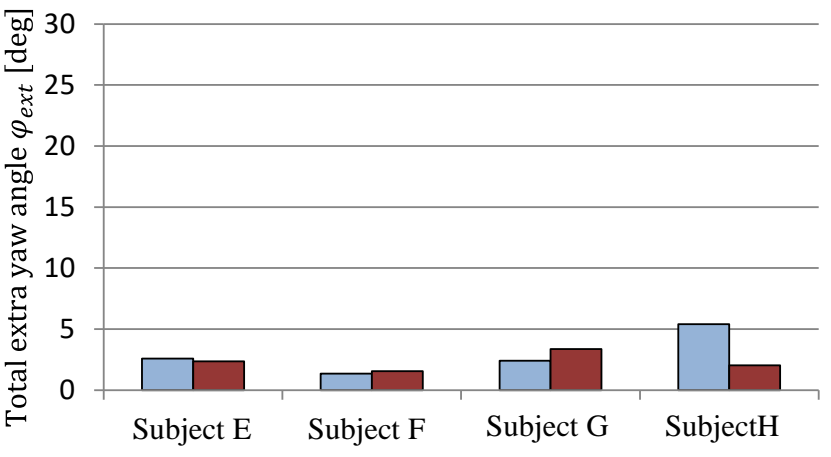

(b) Results for experienced drivers.

Fig. 27 Comparison of total excess yaw angle $\varphi_{\text {ext }}$ between conventional and proposed steering systems. (a) Non-experienced drivers showed large values of total excess yaw angle $\varphi_{\text {ext }}$ when using the conventional steering system. However, the value of total excess yaw angle $\varphi_{\text {ext }}$ was reduced when using the proposed steering system. (b) For experienced drivers, the total excess yaw angle $\varphi_{\text {ext }}$ did not vary greatly between the conventional and proposed steering systems.

\section{Conclusion}

In this study, causes of steering workload during low-speed driving were discussed by measuring and analyzing driver steering actions. A new steering system was also developed and evaluated to make low-speed driving easier.

The study's findings are summarized as follows:

1. At corners where steering grip changes are required, three conditions should be simultaneously satisfied: (1) vehicle motion in a straight line, (2) zero steering wheel angle, and (3) steering wheel grip in neutral position. To achieve these conditions, experience, which a person acquires in a long run, is necessary. Therefore, steering intuitively during low-speed driving is considered to be difficult, as frequent steering grip changes add to the driver's potential workload.

2. In general, if the steering ratio is reduced to make the steering wheel grip change unnecessary, it becomes more difficult to drive due to the reduced steering resolution. However, it was shown that it should be possible to create a steering system that can be controlled easily even with a low steering ratio by changing the driver arm motion and grip of the steering system.

3. It was verified through a lab experiment and test runs that changing the handgrip form of a steering system into a handlebar makes it possible to track the target course accurately without losing stability.

4. Furthermore, it was shown that changing the driver's arm motion during steering to a back-and-forth motion makes the steering system more drivable.

5. The driver steering gain was large and the time constant was small when the drivers used a conventional steering system with a low steering ratio. On the contrary, when the proposed steering system with a handlebar grip was used, the steering gain was kept small and it was possible to steer with good stability.

6. The proposed steering system with a handlebar grip allowed those with no prior car driving experience to steer smoothly and suppressed meandering and instability compared to a conventional steering system with a steering wheel. It was also found that the proposed steering system presents no problems for experienced drivers who drive conventional cars regularly. 


\section{References}

Akamatsu, M., Trends of Automobile Control System From View Point of Driver's Control Behavior, The Society of Automotive Engineers of Japan Symposium, (2011), pp.1-6 (in Japanese).

Isheda, S., Development of an Intelligent Driver Support System, Journal of Society of Automotive Engineers of Japan, 60-12(2006), pp.39-44(in Japanese).

Kahneman,D., Attention and effort, Englewood Cliffs, NJ, Prentice-Hall (1973).

Kakinuma, T., Shino, M., Kamata, M., Proposal on Steering System Based on relationship between Gear Ratio and Reaction Torque Control at Low Speed Region, Proceedings of the Japan Society of Mechanical Engineers -The 15th Transportation and Logistics Conference-(2006), pp.317-320 (in Japanese).

Kitahara, K., Haramiishi, Y., Mouri, H., Proposal of Steering System Considering the Direction of Upper Limb Motion, Transactions of the Japan Society of Mechanical Engineers, Series C,Vol.79, No806 (2013), pp.3327-3334 (in Japanese).

Li, S., Yamabe, S., Sato, Y,. Hirasawa, T., Suda, Y., Driving Feature Extraction Focused on Driver's Steering Behavior in Curving, Transactions of the Society of Automotive Engineers of Japan, 43-6(2012), pp.1387-1392(in Japanese).

Mizuno, T., Hayama, R., Kawahara, S., Research on Relationship between Steering Maneuver and Muscles Activity, JTECT ENGINEERING JOURNAL, No.1010(2012),(in Japanese).

Nagae, N., Kubota, M., Mouri, H., Furushou, H., Investigation of the effect of Steering Effort on the Performance of Driver-Vehicle Closed Loop System, Proceedings of the Japan Society of Mechanical Engineers -Mechanical Engineering Congress, 2006 Japan-, No.06-1 (2006), pp.487-488 (In Japanese).

National police Agency, Traffic accident situation for the fiscal year of H26, available from <http://www.e-stat.go.jp/SG1/estat/List.do?lid=000001132129>, (2013), p.31(In Japanese).

Otsuka, Y., Tachiwana, S., Doi, S., Improvement of Handling Feeling Based on Grasping Characteristic of Driver, Transactions of the Japan Society of Mechanical Engineers, Series C,Vol.79, No806 (2013), pp.3304-3314, (in Japanese).

Sadano, O., Chiku, N., Akutagawa, K., Watanabe, T. and Shimakage, M., Lane-Keeping Support System, Journal of Society of Automotive Engineers of Japan, 55-11(2001), pp.36-41(in Japanese).

Souma, H., Suzuki, K., Wakasugi, T., Hiramastu, K., Analysis of Drivers' Characteristics and Evaluation of Driver Assistance System Using a Driving Simulator, Transaction of International Association of Traffic and Safety Science, Vol.26, No.2 (2001), pp96-102 (in Japanese).

Suzuki, Y., Kazama, K., Haramiishi, Y., Mouri, H., Sugasawa, F., Investigation on the influence of side angle to the driver's handling behavior, Transactions of the Japan Society of Mechanical Engineers, Vol.80, No815 (2014), (in Japanese).

Tajima, T., Sato, K., Tada, Y., Fujita, H., Nakazato, Y., Noguchi, W., Study of the Next Generation Steering System -Study of the Twin Lever Steeling System-, Proceedings of the Society of Automotive Engineers of Japan, No.75-11(2011), pp.7-12(in Japanese).

Tsujimoto, T., Shino, M., Kamata, M., Proposal on New Steering System Based on physical Features of Elderly Driver, Proceedings of the Japan Society of Mechanical Engineers -The 14th Transportation and Logistics Conference-(2005), pp.271-274 (in Japanese). 